This document is confidential and is proprietary to the American Chemical Society and its authors. Do not copy or disclose without written permission. If you have received this item in error, notify the sender and delete all copies.

\title{
Colonization of electrospun polycaprolactone fibers by relevant pathogenic bacterial strains.
}

\begin{tabular}{|r|l|}
\hline Journal: & ACS Applied Materials \& Interfaces \\
\hline Manuscript ID & am-2017-19440d.R1 \\
\hline Manuscript Type: & Article \\
\hline Date Submitted by the Author: & 08-Mar-2018 \\
\hline Complete List of Authors: & $\begin{array}{l}\text { Rumbo, Carlos; Universidad de Burgos, } \\
\text { Tamayo-Ramos, Juan Antonio; Universidad de Burgos - Campus San } \\
\text { Amaro, ICCRAM } \\
\text { Caso, M. Federica; Nanofaber srl. } \\
\text { Rinaldi, Antonio; ENEA and University of L'Auila , UTT-MAT and MEMOCS } \\
\text { Romero-Santacreu, Lorena; International Research Centre in Critical Raw } \\
\text { Materials-ICCRAM, Universidad de Burgos } \\
\text { Quesada, Roberto; Universidad de Burgos, Química } \\
\text { Cuesta-López, Santiago; International Research Centre in Critical Raw } \\
\text { Materials-ICCRAM, Universidad de Burgos }\end{array}$ \\
\hline
\end{tabular}

\section{SCHOLARONE ${ }^{\text {Tw }}$}

Manuscripts 


\title{
Colonization of electrospun polycaprolactone
}

\section{fibers by relevant pathogenic bacterial strains}

Carlos Rumbo ${ }^{1,2, \ddagger} *$, Juan Antonio Tamayo-Ramos ${ }^{1, \ddagger} *$, M. Federica Caso ${ }^{3}$, Antonio Rinaldi $^{4}$, Lorena Romero-Santacreu ${ }^{1}$, Roberto Quesada ${ }^{2}$, and Santiago Cuesta-López ${ }^{1}$ *

1 International Research Centre in Critical Raw Materials-ICCRAM, Universidad de Burgos, Plaza Misael Bañuelos s/n, 09001 Burgos, Spain.

2 Departmento de Química, Facultad of Ciencias, Universidad de Burgos, 09001, Burgos, Spain.

3 Nanofaber srl. Via Anguillarese 301, 00123 Rome, Italy.

4 Italian National Agency for New Technologies, Energy and Sustainable Economic Development (ENEA), Casaccia Research Centre, Via Anguillarese 301, 00123 Rome, Italy.

\begin{abstract}
Electrospun biodegradable polymers have emerged as promising materials for their applications in several fields, including biomedicine and food industry. For this reason, the susceptibility of these materials to be colonized by different pathogens is a critical issue for public health, and their study can provide future knowledge to develop new strategies against bacterial infections. In this work, the ability of three pathogenic bacterial species (Pseudomonas aeruginosa, Acinetobacter baumannii and Listeria monocytogenes) to adhere and form biofilm in electrospun polycaprolactone (PCL) microfibrous meshes was investigated. Bacterial attachment was analyzed in meshes
\end{abstract}


with different microstructure, and comparisons with other materials (borosilicate glass and electrospun polylactic acid (PLA)) fibers were assessed. Analysis included colony forming unit (CFU) counts, scanning electron microscopy (SEM), and crystal violet (CV) staining. All the obtained data suggest that PCL meshes, regardless of their microstructure, are highly susceptible to be colonized by the pathogenic relevant bacteria used in this study, so a pretreatment or a functionalization with compounds that present some antimicrobial activity or antibiofilm properties is highly recommended before their application. Moreover, an experiment designed to simulate a chronic wound environment was used to demonstrate the ability of these meshes to detach biofilms from the substratum where they have developed, thus making them promising candidates to be used in wound cleaning and disinfection.

KEYWORDS: Electrospun polycaprolactone, microfibers, nosocomial pathogens, foodborne pathogens, bacterial attachment, biofilm.

\section{INTRODUCTION}

The use of biodegradable polymers as biomaterials in medical equipment and hospital settings set a major advance in modern medicine, being the main advantage their capacity to be degraded, excreted or reabsorbed in the organism, thus avoiding surgical removal. A wide range of natural and synthetic biodegradable polymers are being used in biomedicine, with a great variety of medical applications. ${ }^{1}$ Tissue engineering is one of the main fields where these polymers are implemented. The production of threedimensional scaffolds provides a space with an structure similar to the extracellular matrix, which is appropriate for cell adhesion and proliferation, playing a crucial role in new tissue regeneration. ${ }^{2}$ Many advanced procedures have been developed to obtain these scaffolds. ${ }^{3}$ Among them, electrospinning is one of the most successful techniques. 
Through this method, fibers in the nanoscale or microscale range are obtained, with the possibility of controlling morphology, diameter and pattering of electrospun nanofibers over a wide range value. ${ }^{4}$ Polycaprolactone (PCL), a synthetic biodegradable and semicrystaline polyester, is one of the most suitable materials for use in tissue engineering. Several studies have demonstrated that electrospun PCL scaffolds can be efficiently applied in different fields, such as bone regeneration, ${ }^{5,6}$ cardio-vascular tissue engineering ${ }^{7,8}$ or skin tissue engineering. ${ }^{9,}{ }^{10}$ Moreover, the use of PCL in combination with other compounds, such as nanoparticles or hyaluronic acid, has been shown to be a safe and effective wound dressing. ${ }^{11,12}$

Hospital-acquired infections caused by multidrug-resistant bacteria are one of the most important problem that modern medicine is facing nowadays, presenting incidence rates of $5 \%$ and $7.1 \%$ in the United States and the European Union respectively in $2013 .{ }^{13}$ Bacteria can attach to biomaterials and proliferate forming biofilms, which can cause implant related infections. ${ }^{14}$ Biofilms are microorganism associations, formed by one or more species, attached to a substratum and embedded in a complex self-produced matrix of polymeric substances, being the main components exopolysaccharides, proteins and nucleic acids. ${ }^{15,16}$ This structure confers protection to the microorganisms contained in it. Compared to their planktonic states, biofilm-embedded bacteria are significantly more resistant to desiccation, environmental stress, and ultraviolet (UV) light exposure, as well as to ordinary antibiotics and disinfectants. ${ }^{17}$ All of this turns biofilms into a serious risk in medicine. Biofilm formation is frequent in clinical strains and plays an important role in chronic colonization of human tissues and their persistence in implanted medical devices. ${ }^{18,} 19$ For all these reasons, investigating biofilm formation on biomaterials is a crucial issue for modern medicine. 
Beyond the biomedical field, there is a growing interest in the utilization of electrospun biodegradable polymers in the food industry, due to the many possible applications, such as encapsulation of food materials, and their use in packaging or as processing aids. $^{20,21}$ However, most of the foodborne pathogens, such as Listeria monocytogenes, Salmonella enterica or Escherichia coli, can manage to colonize different materials and surfaces present in food processing plants through the development of biofilm ${ }^{22}$, being this an important public health issue. In fact, as with the biomedical field, microbial biofilms are a problematic and persistent issue in the food industry, where they have been involved in many foodborne outbrakes. ${ }^{23}$

Bacterial attachment and biofilm formation in abiotic surfaces is a complex process that, besides bacterial surface characteristics, is critically affected by surface topography. Substratum properties such as morphology, surface chemistry, roughness, material surface energy, wettability, or zeta potential influence the colonization of a biomaterial. ${ }^{24,25}$ Despite the critical importance of biofilms for public health, and the growing use of electrospun microfibers in several fields, to our knowledge, there are only a few studies focusing on microbial adhesion, colonization and biofilm formation on these structures. The analysis of interactions between bacteria and electrospun micro/nanofibers is relevant to develop new strategies to prevent the occurrence of associated infections, and to determine new potential applications of these novel materials. Kargar et al. investigated the interactions of bacteria with fibrous substrates, finding that the adhesion of Pseudomonas aeruginosa on polystyrene (PS) fibers is dependent on fiber diameter and spacing. ${ }^{26}$ Abrigo et al. observed that the diameter of the fibers influences the ability of E. coli, P. aeruginosa and Staphylococcus aureus to proliferate and colonize electrospun PS fibers. ${ }^{27}$ By the same token, these authors 
described the effect of surface chemistry in the attachment and proliferation onto electrospun PS fibers. ${ }^{28}$

In this study, the ability of three pathogenic species belonging to different environments to adhere to electrospun PCL microfiber meshes and form biofilms was analyzed and compared using different assays. Two of the most important Gram-negative nosocomial pathogens, $P$. aeruginosa and Acinetobacter baumannii, with the capacity to acquire and develop multiple resistance mechanisms ${ }^{29,}{ }^{30}$ were used in this study. In addition, the ability to form biofilm in these microfibers was studied in L. monocytogenes, one of the most important foodborne pathogens. ${ }^{31}$ The attachment, the structure and the viability of the biofilms formed on these meshes were studied using crystal violet (CV) staining, scanning electron microscope (SEM) and colony forming unit (CFU) quantification. Also, since $P$. aeruginosa and A. baumannii are frequently involved in wound infections, ${ }^{32}$ bacterial transfer experiments using PCL meshes in direct contact with bacteria previously grown in agar plates were performed to simulate a wound environment. Furthermore, CFU count assays were carried out to study the influence of the PCL mesh microstructure on bacterial attachment, and to perform comparative experiments with borosilicate glass and electrospun polylactic acid (PLA) fibers, materials commonly used in hospital environments and biomedicine applications respectively.

\section{MATERIALS AND METHODS}

\section{Materials and specimen preparation}

All PCL microfiber meshes used in this study (NBARE, Pro3 and Pro4) were supplied by Nanofaber S.R.L. All of them were synthesized using the linear thermoplastic PCL diol polymer CAPA® 6800 (80,000 MW) (Prestor), chloroform (99.2\% purity, 
stabilized with $0.6 \%$ ethanol; VWR) and dimethylformamide (DMF) (100\% purity; VWR). PCL was dissolved to obtain $12 \%$ (w/w) solutions in $40 \mathrm{~mL}$ DMF/chloroform 35:65 (NBARE), $40 \mathrm{~mL} \mathrm{DMF/chloroform} \mathrm{20:80} \mathrm{(Pro3)} \mathrm{or} 40 \mathrm{~mL}$ chloroform (Pro4). Table 1 shows the values of the electrospinning parameters that were used for each fiber type. The electrospun material was deposited on a flat aluminium collector. NBARE meshes are formed by 2 kinds of microfiber with different size, while Pro3 and Pro4 meshes present a uniform microfiber size (Table 1). PLA fiber meshes were supplied by Nanopharma a. s. Round dishes of all biomaterials were obtained with a round punch with a diameter of $12 \mathrm{~mm}$. Borosilicate glass coverslips of $12 \mathrm{~mm}$ in diameter (Thermo Scientific Menzel) were purchased from Fisher Scientific. Before use, all materials were sterilized by immersion in $70 \%$ ethanol (v/v) for 30 minutes, and UV irradiation for 30 minutes.

Table 1. Parameters used for the electrospinning of PCL solutions and microfiber diameter in the different fiber types.

\begin{tabular}{ccccccccc}
\hline fiber type & $\begin{array}{c}\text { flow rate } \\
(\mu \mathrm{L} / \mathrm{h})\end{array}$ & $\begin{array}{c}\text { applied } \\
\text { voltage } \\
(\mathrm{kV})\end{array}$ & $\begin{array}{c}\text { Moving } \\
\text { needle } \\
\text { diameter } \\
(\mathrm{mm})\end{array}$ & $\begin{array}{c}\mathrm{X} \text { axis } \\
\text { speed } \\
(\mathrm{mm} / \mathrm{s})\end{array}$ & $\begin{array}{c}\text { Y axis } \\
\text { speed } \\
(\mathrm{mm} / \mathrm{s})\end{array}$ & $\begin{array}{c}\text { spinning } \\
\text { distance } \\
(\mathrm{cm})\end{array}$ & $\begin{array}{c}\text { deposition } \\
\text { time }(\mathrm{min})\end{array}$ & $\begin{array}{c}\text { microfiber } \\
\text { diameter } \\
(\mu \mathrm{m})\end{array}$ \\
\hline NBARE & 6000 & 23 & 1.7 & 60 & 6 & 24 & 60 & $2.19 \pm 0.13$ \\
Pro3 & 4000 & 29 & 1.7 & 60 & 6 & 24 & 60 & $2.21 \pm 0.48$ \\
Pro4 & 4000 & 23 & 1.7 & 60 & 6 & 24 & 60 & $6.43 \pm 1$ \\
\hline
\end{tabular}

\section{Bacterial strains and culture conditions}

The bacterial strains A. baumannii ATCC 19606, P. aeruginosa PAO1 and $L$. monocytogenes NCTC 11994 were purchased from Spanish Type Culture Collection. A. baumannii and $P$. aeruginosa were maintained at $30^{\circ} \mathrm{C}$ in Luria-Bertani (LB) broth or agar. L. monocytogenes was maintained in Tryptic Soy broth (TSB) or agar at $30^{\circ} \mathrm{C}$. 


\section{Bacterial count determination}

To study the number of bacteria attached to the different materials, one colony of each strain grown on agar media was inoculated into $5 \mathrm{~mL}$ of liquid media and incubated overnight. Then, a dilution 1:100 of the overnight culture was dispensed into each well of a 24 well plate containing materials, and incubated at $30^{\circ} \mathrm{C}$ for 48 hours under static conditions. The materials were then rinsed three times with Dulbecco's PhosphateBuffered Saline (DPBS) to remove any unbound bacteria, and transferred into sterile conical tubes containing $5 \mathrm{~mL}$ of DPBS. The tubes were vortexed at full speed for 1 minute and then placed in an ultrasonic bath and sonicated for 15 minutes at low power intensity, to release the attached bacteria from the material. After an additional vortex step, suspensions were serially diluted with DPBS and cultured overnight. The CFUs were counted to determine the number of viable adherent bacteria, and the Log base 10 of the CFUs $/ \mathrm{cm}^{2}$ (Log CFUs $/ \mathrm{cm}^{2}$ ) was calculated. Each assay included at least three independent replicates.

\section{Scanning electron microscopy}

Biofilms of each strain were grown under the same conditions as those explained above. Once microfiber meshes were washed 3 times, they were fixed with $2 \%$ paraformaldehyde, $2 \%$ glutaraldehyde, $3 \mathrm{mM} \mathrm{CaCl}_{2}$ in DPBS, and sequentially dehydrated for $10 \mathrm{~min}$ in 50, 70, 80, 90, 95 and 100\% (x2) (v/v) ethanol. Finally, the meshes were coated with a layer of gold and examined with environmental scanning electron microscope (FEI-Quanta 200F).

Electrospun meshes NBARE, Pro3 and Pro4 were previously coated with gold (15-30 $\mathrm{nm}$ ) using the Edwards Sputter/Coater S150B deposition chamber and observed and analyzed with scanning electron microscope (Leo 1530, ZEISS, Germany). 


\section{Crystal Violet assay}

Biofilms of each strain were grown under the same conditions as those explained above. After the washing step with DPBS, meshes were transferred into clean wells, and stained with a 1:2 dilution of 1\% (w/v) crystal violet solution (Sigma-Aldrich) for 30 minutes at room temperature. The excess stain was washed 3 times with DPBS, and membranes were transferred again to clean wells and photographed. All experiments were carried out in triplicate.

\section{Agar-mesh bacterial transfer experiments}

Bacterial transfer experiments were performed according to a method described previously by Abrigo et al. ${ }^{27}$ In brief, one colony of each strain grown on agar media was inoculated into $5 \mathrm{~mL}$ of liquid media and incubated overnight. The obtained culture was diluted up to an optical density at $600 \mathrm{~nm}\left(\mathrm{OD}_{600}\right)$ of 0.3 in clean media, and 100 $\mu \mathrm{L}$ of the suspension was spread onto agar plates, which were incubated $18 \mathrm{~h}$ at $30^{\circ} \mathrm{C}$ to obtain a confluent biofilm. Subsequently, microfiber meshes were placed on the agar in contact with the biofilm, and the plates were incubated $1 \mathrm{~h}$ at $30^{\circ} \mathrm{C}$. After incubation, meshes were removed from the plate, and the culture was checked for the presence of spots.

\section{Statistical analysis}

Statistical analysis data are presented as means \pm SD. Differences between the attachment in PCL microfiber meshes and other materials were stablished using a Student's t-test. The one-way analysis of variance (ANOVA) was used for multiple comparisons, followed by Tukey post hoc test. Statistical tests were carried out using 
Prism 6.0 (GraphPad Prism, GraphPad Software, Inc.). Differences were considered significant at $P \leq 0.05$.

\section{RESULTS AND DISCUSSION}

\section{Determination of biofilm formation in PCL microfiber meshes by different pathogenic strains.}

Microbial biofilms are formed by a matrix of extracellular polymeric substances that allow bacteria to resist chemical and physical stress. ${ }^{33}$ Biofilms have a high impact in industrial and clinical settings, representing a significant concern for human health. In this study, the susceptibility of commercial electrospun PCL microfiber meshes specially designed for use in biomedicine, named as NBARE, to be colonized by different bacterial pathogens has been analyzed. For this purpose, different methodologies were applied. Figure 1 shows the structure of NBARE fibers. This mesh type consists in two kinds of fiber with different size, one with an average fiber thickness of $2.19 \mu \mathrm{m}$, and the other with an average fiber thickness of $0.80 \mu \mathrm{m}$.
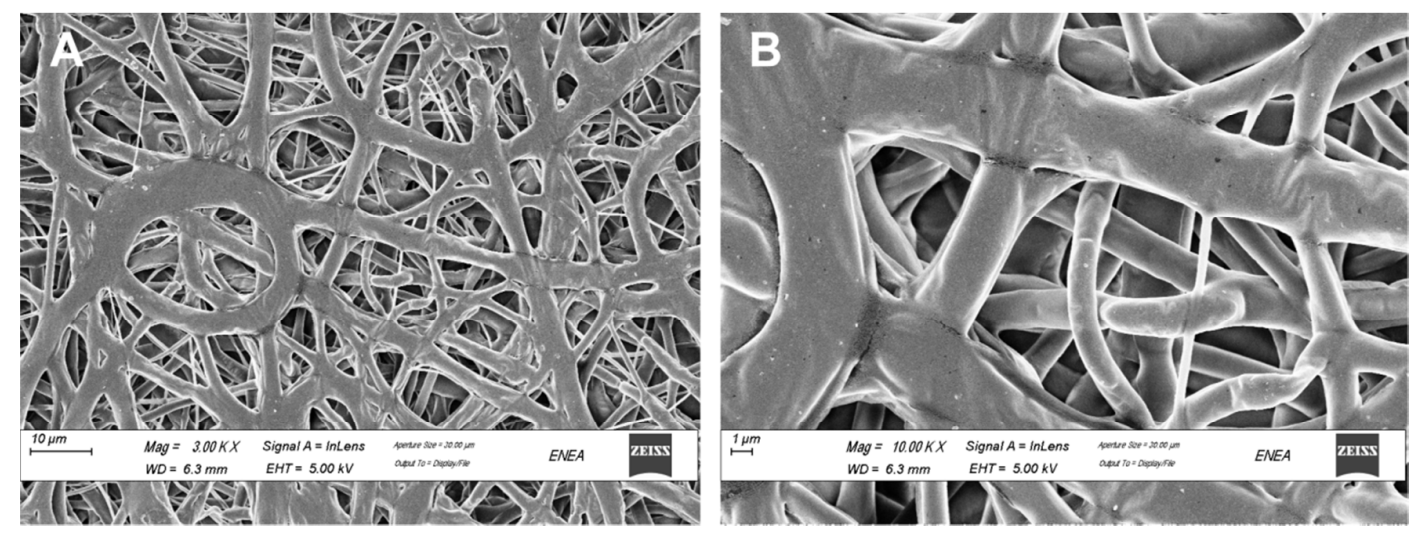

Figure 1: SEM image showing the microstructure of NBARE fibers. Overview (A) and detail (B). Image A: Original magnification $\times 3000($ Scale bar $=10 \mu \mathrm{m})$; Image B: Original magnification $\times 10000($ Scale bar $=1 \mu \mathrm{m})$ 
To investigate the ability of the different pathogenic species to colonize PCL microfibers, meshes were exposed to liquid cultures of the strains under study during 48 h. After this incubation period, meshes were washed and adhered bacteria were detached by vortexing and sonication. CFU counts were used to analyze the number of viable bacteria attached. The results obtained are represented in Figure 2. P. aeruginosa and L. monocytogenes had similar Log CFUs $/ \mathrm{cm}^{2}$ attached to PCL microfibers, while $A$. baumannii presented a statistically significant reduction of attached bacteria in comparison with the two other strains $(P \leq 0.001)$, presenting a bacterial load of around 1 $\log$ unit lower than the others.

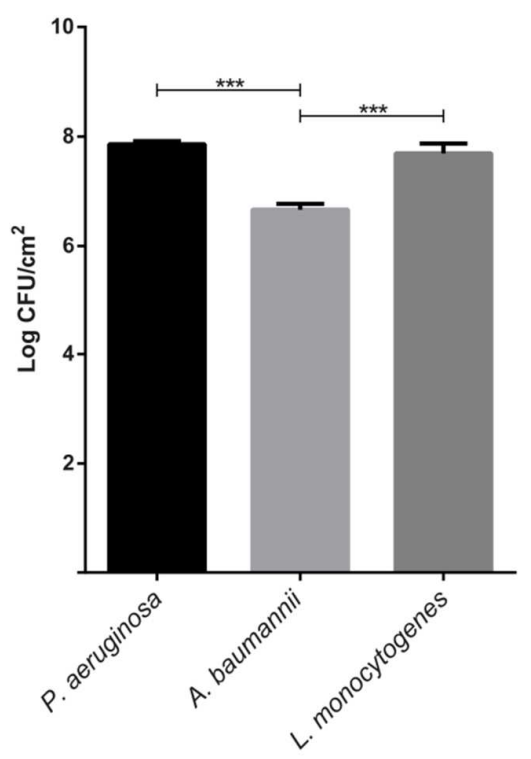

Figure 2: Ability of different bacterial strains to attach to PCL fibers expressed as Log $\left(\mathrm{CFU} / \mathrm{cm}^{2}\right)$. Data represent the mean $( \pm$ standard deviation, $\mathrm{SD})$ of three independent replicates. Differences were stablished using a One-way ANOVA, and considered significant at $P \leq 0.05$. *** $P \leq 0.001$.

Biofilms were also analyzed using SEM (Figure 3). We observed that P. aeruginosa (Figure 3A, B, C) and L. monocytogenes (Figure 3G, H, I) showed high ability to form biofilms in PCL microfibers. In both cases, the microbial biofilms spread across the 
mesh, being able to form continuous layers over the microfiber nets. These results are consistent with those obtained in CFU counts, which showed very high values of bacteria $/ \mathrm{cm}^{2}$. However, the images obtained for A. baumannii are intriguing (Figure 3D, E, F), since we could not find a clear evidence of the attachment of this bacteria to PCL microfibers through the SEM analysis. CFU data corresponding to the attachment analysis of this strain suggested that it has the ability to form biofilms into electrospun surface. Therefore, it could be possible that the sample preparation protocol for SEM analysis alters the weakly formed biofilm, causing a massive detachment.

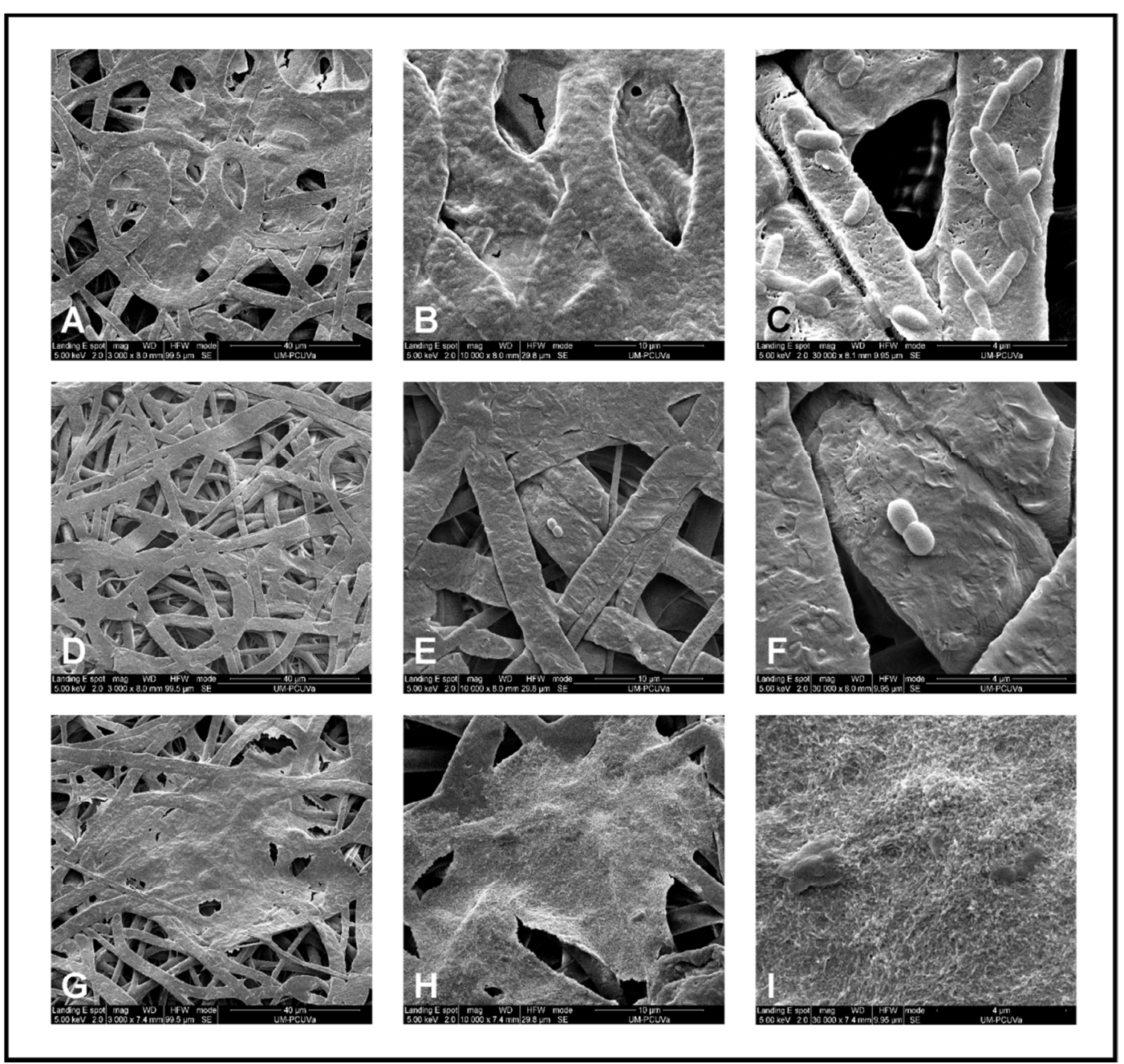

Figure 3: SEM images showing bacterial biofilms of different pathogenic strains formed on PCL fibers. Biofilms formed by P. aeruginosa (A, B, C); A. baumannii (D, 
$\mathrm{E}, \mathrm{F})$ and L. monocytogenes $(\mathrm{G}, \mathrm{H}, \mathrm{I})$. Images $\mathrm{A}, \mathrm{D}$ and $\mathrm{G}$ : Original magnification $\times 3000($ Scale bar $=40 \mu \mathrm{m})$; Images B, E and F: Original magnification $\times 10000$ (Scale bar $=10 \mu \mathrm{m})$; Images G, H and I: Original magnification $\times 30000($ Scale bar $=4 \mu \mathrm{m})$.

A qualitative analysis of the bacterial attachment to the PCL meshes was also performed using crystal violet staining (Figure 4). Crystal violet stains the whole biofilm biomass, including cells and extracellular polymeric matrix. ${ }^{34}$ As expected after the results obtained in the CFU determination and SEM experiments, all meshes appeared completely stained. Depending on the bacterial culture put in contact with the electrospun polymeric surface, different intensities of the stain could be distinguished, suggesting differences in the biomass content of the biofilms attached to them. Meshes incubated with $P$. aeruginosa (Figure 4B) presented the most intense color, followed by the meshes incubated with A. baumannii and L. monocytogenes (Figure 4C and E respectively), indicating that biofilms formed by this strain presented the higher amount of biomass. Control meshes incubated with culture media without bacteria were not stained by CV (Figure 4A and D). Remarkably, the PCL microfiber meshes incubated with $A$. baumannii showed to be well stained by the crystal violet reagent, indicating the formation of a biofilm by this bacterial specie. This result is coherent with the CFU quantification results obtained for this strain, but not with our observation through SEM analysis. In this regard, as suggested above, it may well be that sample preparation protocol for SEM analysis caused complete bacterial detachment. 
Figure 4: CV staining of biofilms onto PCL microfiber meshes. A: Control meshes incubated with LB; B: Meshes incubated with $P$. aeruginosa; C: Meshes incubated with A. baumannii; D: Control meshes incubated with TSB; E: Meshes incubated with $L$. monocytogenes.

\section{Ability of electrospun PCL microfiber meshes to detach preformed biofilms from the substrate.}

PCL presents good characteristics such as biocompatibility, biodegradability, and great strength properties, which make this material appropriate to be used as wound dressing. ${ }^{12}$ For this reason, we found interesting to examine the ability of the selected bacterial strains to attach to the PCL microfibers in a wound dressing experiment following the method described by Abrigo et al. ${ }^{27}$ Through this method, by putting PCL meshes in direct contact with confluent biofilms grown in agar plates, a realistic model that simulates a wound bed contaminated by bacteria is obtained, and the capacity of the meshes to attract and remove the bacterial cells can be studied. P. aeruginosa and $A$. baumannii are frequently involved in wound infections. ${ }^{32}$ L. monocytogenes was also included in this experiment in spite of the fact that wound infections caused by this 
pathogen are uncommon. Figure 5 shows agar cultures where the mesh was in contact with biofilms for 1 hour and removed afterwards. As shown in this figure, it can be clearly distinguished where the meshes were placed, as the bacterial biomass present under them was almost completely removed in all cases. In a deep examination of the plates, it was observed that the spots left by P. aeruginosa and L. monocytogenes (Figure 5A, C) were almost clean and free of remains of culture, which suggests that these strains attached very efficiently to the meshes. In case of $A$. baumannii (Figure 5B), remnants of the biofilm could be observed in the spots left once the PCL meshes were removed, which indicates that this pathogen has a lower ability to attach to this material. The obtained results are in concordance with those observed in CFU determination experiments, where $A$. baumannii was the strain that presented the lowest number of attached bacteria to PCL meshes. Nevertheless, the obtained results suggest that electrospun PCL microfibers could be used to attach and significantly remove the bacteria present in the surface of a wound.
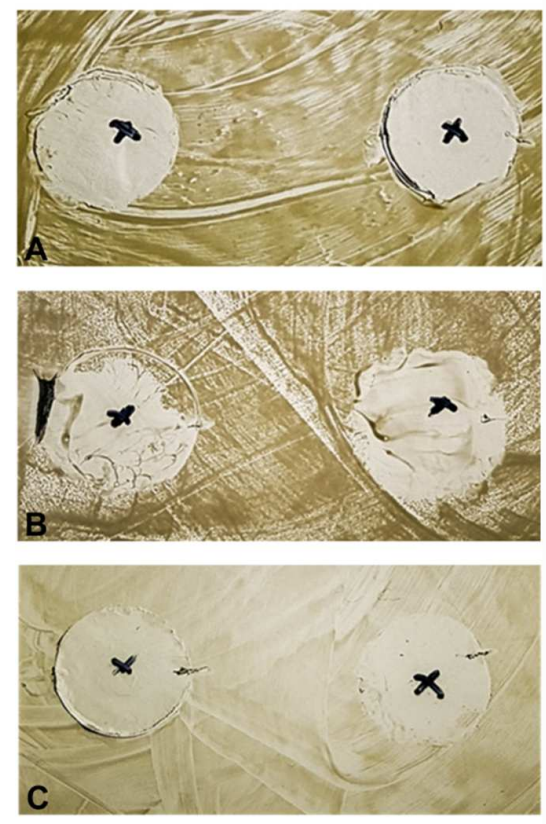
Figure 5: Agar culture after mesh removal: A: P. aeruginosa; B: A. baumannii; C: $L$. monocytogenes.

\section{Impact of different electrospun PCL mesh microstructure on bacterial attachment.}

In order to study if the ability of the selected bacterial strains to adhere to the PCL meshes is affected by the microstructure, two types of meshes with clear microstructural differences and different microfiber diameter, named as Pro3 and Pro4, were analyzed using CFU counts and SEM. In Figure 6 the structure of both types of these fibers is represented. Pro3 microfiber meshes (Figure 6A) were designed with an average fiber thickness of $2.21 \mu \mathrm{m}$, while Pro4 fiber meshes (Figure 6B) were designed with an average fiber thickness of $6.43 \mu \mathrm{m}$.
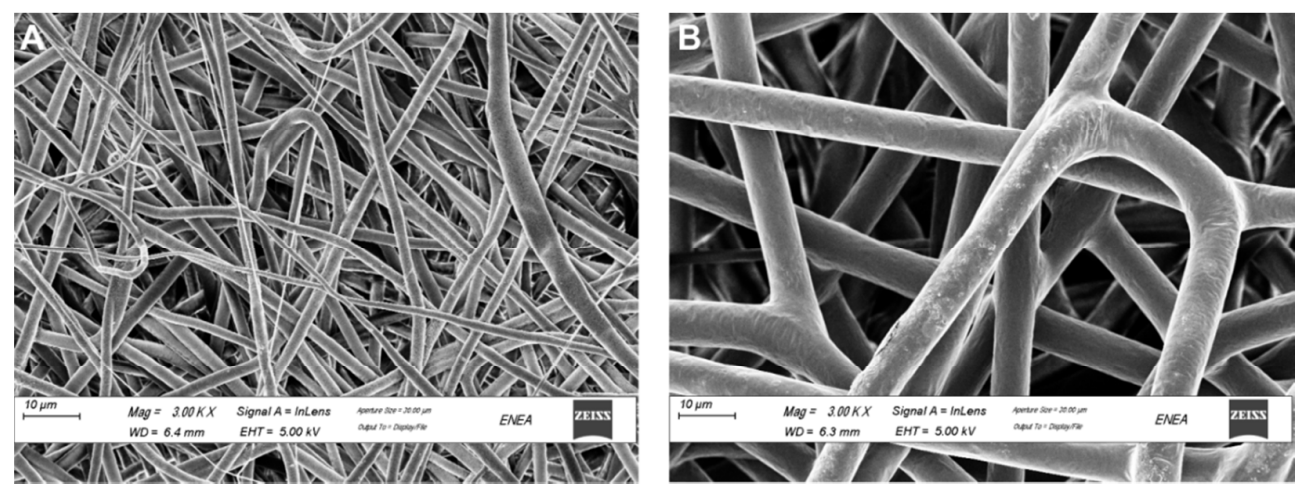

Figure 6: SEM images showing the microstructure of Pro3 (A) and Pro4 PCL fibers (B). Differences between both types are clearly distinguishable. Original magnification $\times 3000$ (Scale bar $=10 \mu \mathrm{m})$.

Figure 7 displays the results obtained in CFU determinations corresponding to the biofilm formation of $P$. aeruginosa, A. baumannii and L. monocytogenes on the microfibers Pro3 and Pro4. In case of A. baumannii, bacterial counts obtained showed 
that a higher number of bacteria were attached to the Pro3 material when compared to the Pro4, but the differences obtained were not statistically significant (Figure 7b). $P$. aeruginosa and L. monocytogenes presented a similar number of bacterial attachment to both fiber types, suggesting that their ability to form biofilms on PCL microfibers is not easily altered by the material microstructure (Figures $7 \mathrm{a}, \mathrm{c}$ ).

a)

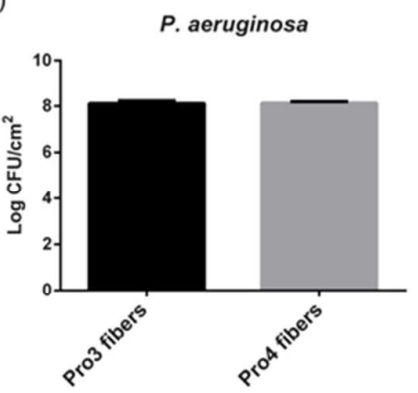

b)

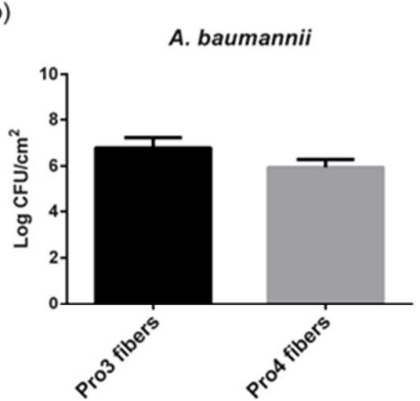

c)

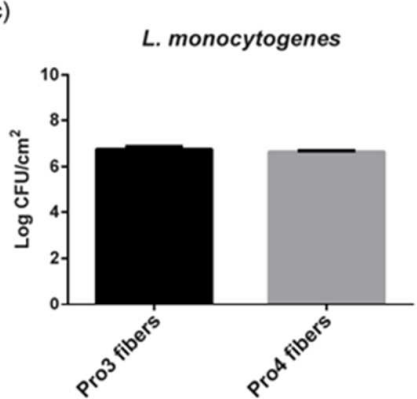

Figure 7: Bacterial attachment $\left(\log \mathrm{CFUs} / \mathrm{cm}^{2}\right)$ to Pro3 fibers (black bars) and Pro4 fibers (Grey bars. a): P. aeruginosa ; b): A. baumannii; c): L. monocytogenes. Data represent the mean of three independent replicates ( \pm standard deviation, $\mathrm{SD})$.

To illustrate biofilm formation in both kinds of meshes, $P$. aeruginosa, the pathogen that showed the highest ability to colonize PCL microfibers, was selected to perform SEM experiments (Figure 8). As expected, and in line with the CFU counts, both Pro3 and Pro4 presented biofilms spread across the mesh, appearing areas completely covered. 

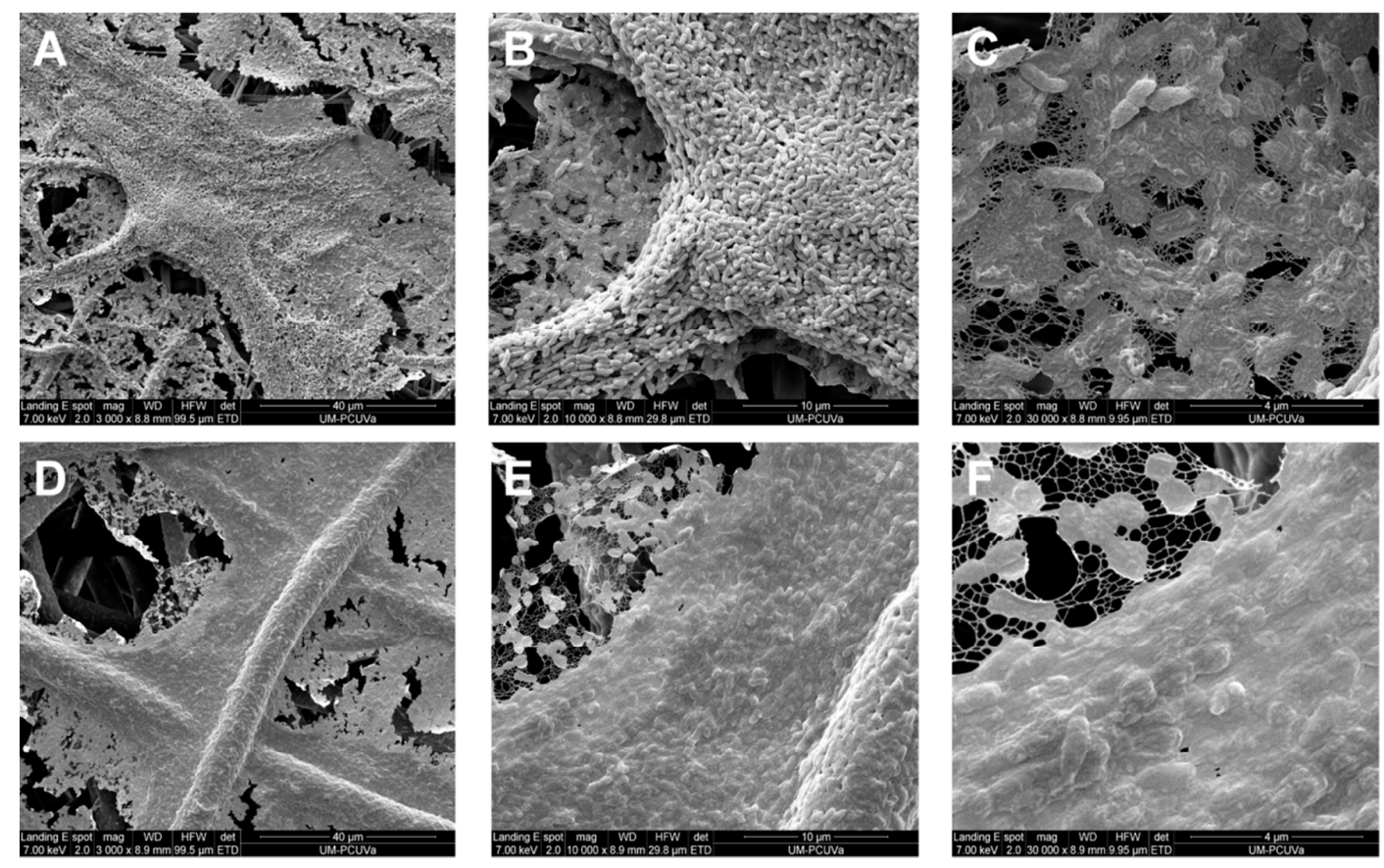

Figure 8: SEM images showing bacterial biofilms of $P$. aeruginosa formed on Pro3 (A, B, C) and Pro4 fibers (D, E, F). A and D: Original magnification $\times 3000$ (Scale bar $=40$ $\mu \mathrm{m})$; Images $\mathrm{B}$ and E: Original magnification $\times 10000($ Scale bar $=10 \mu \mathrm{m})$; Images $\mathrm{C}$ and F: Original magnification $\times 30000($ Scale bar $=4 \mu \mathrm{m})$.

Previous studies have shown that fiber diameter and spacing influence the attachment to electrospun PS of some pathogenic species. ${ }^{26,27}$ Abrigo et al. concluded that meshes with an average fiber diameter close to bacterial size were the best support for bacterial adhesion. ${ }^{27}$ In our study, changes in the bacterial load between meshes with different microstructure and microfiber diameter were not observed. In the present study, the microfibers had, in all cases, a diameter higher than that of the selected bacteria, and incubation time of the liquid cultures was much longer (48 hours) than that used by Abrigo et al. (1 hour). Therefore, both studies are not clearly comparable, as they focus on different stages of bacterial colonization. 


\section{Comparative analysis of bacterial colonization on different materials applied in the food and biomedical fields}

The pathogenic strains selected have shown good ability to colonize different electrospun PCL surfaces under the specific experimental conditions used. At this point, we decided to study their colonizing ability in other materials used in medical and food applications as well. Borosilicate glass and electrospun PLA microfiber meshes were selected. Borosilicate glass can be found in hospital environments, being part of medical devices, and coverslips made of this material are commonly used in research studies that involve the development of microbial biofilms. PLA is a thermoplastic and biodegradable aliphatic polyester derived from lactic acid that presents an excellent biocompatibility and mechanical properties, which makes it highly suitable for its use in a wide spectrum of applications in the medical field and in food packaging. The same experimental approach was used. Therefore, CFU counts were carried out on PCL (NBARE meshes), PLA and glass surfaces, and the obtained results were compared. The number of viable bacteria attached to borosilicate glass in comparison to PCL (Log CFUs $/ \mathrm{cm}^{2}$ ) is shown in Figure 9. Clear differences could be observed between both surfaces. In all cases a statistically significant reduction in the number of bacteria attached to glass coverslips was observed. The colonization of PCL fibers was found between 1 and $3 \log$ units higher than that of borosilicate glass coverslips in $P$. aeruginosa, A. baumannii and L. monocytogenes. 
a)

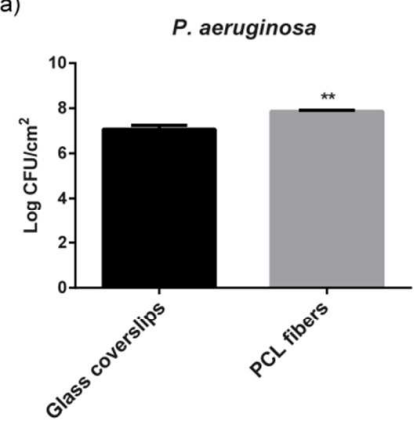

b)

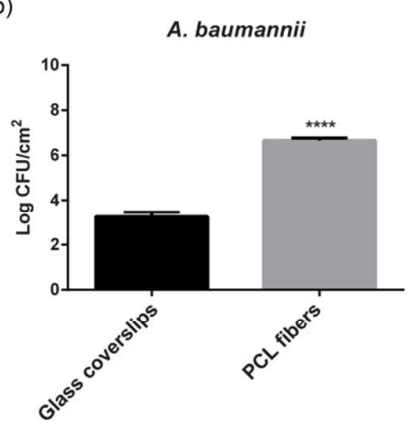

c)

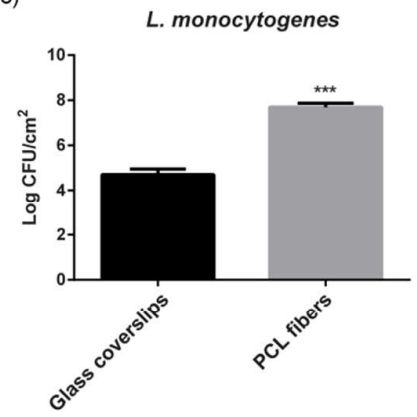

Figure 9: Bacterial attachment (Log $\mathrm{CFUs} / \mathrm{cm}^{2}$ ) to borosilicate glass coverslips (black bars) and electrospun PCL meshes (Grey bars). a): P. aeruginosa; b): A. baumannii; c): L. monocytogenes. Data represent the mean of three independent replicates $( \pm$ standard deviation, SD). Differences were stablished using a Student's t-test, and considered significant at $\mathrm{P} \leq 0.05$. $* * \mathrm{P} \leq 0.01, * * * \mathrm{P} \leq 0.001, * * * * \mathrm{P} \leq 0.0001$.

In order to study whether the attachment of the pathogenic strains to electrospun materials is influenced by their composition, the bacterial attachment in commercial PLA meshes was studied and compared with the PCL meshes. The viable bacterial counts corresponding to the biofilm formation in both fiber types is represented in Figure 10. PCL fibers seem to be more likely to be colonized by $A$. baumannii than PLA fibers (Figure 10b), but the observed differences were not statistically significant. In the case of L. monocytogenes and $P$. aeruginosa, very similar levels of colonization were observed in both types of fibers (Figure 10a, c). The results obtained in these comparative studies revealed that the scaffold provided by electrospun microfibers facilitates the attachment and proliferation of bacteria, being these structures more suitable to be colonized than borosilicate glass, whose characteristics such as its smooth surface and its hydrophilicity make this material surface more difficult to be colonized by pathogens. ${ }^{35}$ 
a)

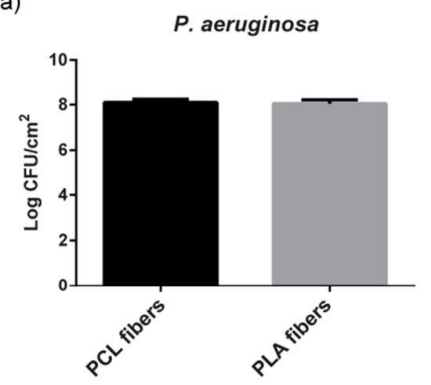

b)

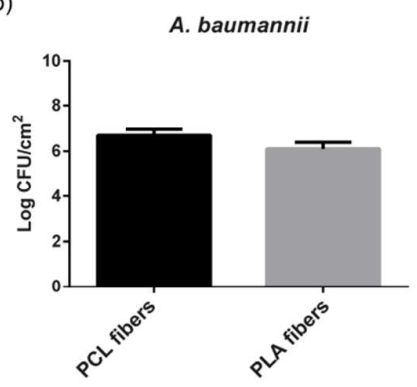

c)

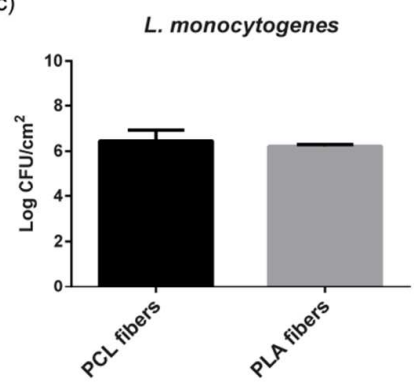

Figure 10: Bacterial attachment $\left(\log \mathrm{CFUs} / \mathrm{cm}^{2}\right)$ to electrospun PCL meshes (black bars) and electrospun PLA meshes (Grey bars) a): P. aeruginosa; b): A. baumannii; c):

L. monocytogenes. Data represent three independent replicates $( \pm$ standard deviation, $\mathrm{SD})$.

\section{CONCLUSION}

Electrospun PCL microfiber meshes showed to be highly susceptible to be colonized by different pathogenic bacteria, such as $P$. aeruginosa, $A$. baumannii and $L$. monocytogenes, important pathogens involved in nosocomial infections and foodborne outbreaks respectively. SEM and CV experiments indicated that biofilms spread across the mesh, and the microbial biomass is uniformly dispersed along them. PCL and PLA microfiber meshes are better substrata for bacterial adhesion than other materials such as borosilicate glass, suggesting that the $3 \mathrm{D}$ structure of electrospun microfibers constitutes a an adequate support for bacterial attachment and proliferation. In this regard, we have also observed that the microfiber microstructure of the electrospun PCL meshes do not seem to influence the bacterial attachment in the species studied after long incubation times. Furthermore, the ability of the PCL meshes to detach biofilms from the substrate, as it was observed in the bacterial transfer experiments, opens the possibility of using this material as an efficient tool for cleaning and disinfecting wounds. The presented results suggest the relevance of functionalizing electrospun 
microfibers with antimicrobial or antibiofilm agents prior their use in biomedicine or food industry applications to prevent their colonization by undesired pathogenic species.

\section{AUTHOR INFORMATION}

\section{Corresponding Authors}

*Carlos Rumbo: E-mail: crumbo@ubu.es

*Juan Antonio Tamayo-Ramos. E-mail: ja.tamayoramos@gmail.com

*Santiago Cuesta López: E-mail: scuestalopez@gmail.com

\section{Author Contributions \\ †These authors contributed equally.}

\section{Notes:}

The authors A. Rinaldi and F. Caso are affiliated with the company NANOFABER srl (www.nanofaber.com).

\section{ACKNOWLEDGEMENTS}

This research work has been developed at the Consolidated Research Unit UIC-154. The Project has received funding from the European Union's H2020 research and innovation programme under the Marie Skłodowska-Curie grant agreement No 691095 and Junta de Castilla y Leon-FEDER (projects BU079U16 and BU092U16). JATR and CRL thank Junta de Castilla y León and FEDER for a postdoctoral contract. 


\section{REFERENCES}

(1) Ulery, B. D.; Nair, L. S.; Laurencin, C. T., Biomedical Applications of Biodegradable Polymers. J. Polym. Sci. B. Polym. Phys. 2011, 49, 832-864.

(2) Wei, G.; Ma, P. X., Nanostructured Biomaterials for Regeneration. Adv. Funct. Mater. 2008, 18, 3566-3582.

(3) Lu, T.; Li, Y.; Chen, T., Techniques for fabrication and construction of threedimensional scaffolds for tissue engineering. Int. J. Nanomedicine. 2013, 8, 337-350.

(4) Ribba, L.; Parisi, M.; D'Accorso, N. B.; Goyanes, S., Electrospun nanofibrous mats: from vascular repair to osteointegration. J. Biomed. Nanotechnol. 2014, 10,3508-3535.

(5) Son, S. R.; Linh, N. B.; Yang, H. M.; Lee, B. T., In vitro and in vivo evaluation of electrospun PCL/PMMA fibrous scaffolds for bone regeneration. Sci. Technol. Adv. Mater. 2013, 14, 015009.

(6) Heydari, Z.; Mohebbi-Kalhori, D.; Afarani, M. S., Engineered electrospun polycaprolactone (PCL)/octacalcium phosphate (OCP) scaffold for bone tissue engineering. Mater. Sci. Eng. C. Mater. Biol. Appl. 2017, 81, 127-132.

(7) Reddy, C. S.; Venugopal, J. R.; Ramakrishna, S.; Zussman, E., Polycaprolactone/oligomer compound scaffolds for cardiac tissue engineering. J. Biomed. Mater. Res. A. 2014, 102, 3713-3725.

(8) Fu, W.; Liu, Z.; Feng, B.; Hu, R.; He, X.; Wang, H.; Yin, M.; Huang, H.; Zhang, H.; Wang, W., Electrospun gelatin/PCL and collagen/PLCL scaffolds for vascular tissue engineering. Int. J. Nanomedicine. 2014, 9, 2335-2344.

(9) Lou, T.; Leung, M.; Wang, X.; Chang, J. Y.; Tsao, C. T.; Sham, J. G.; Edmondson, D.; Zhang, M., Bi-layer scaffold of chitosan/PCL-nanofibrous mat and PLLA-microporous disc for skin tissue engineering. J. Biomed. Nanotechnol. 2014, 10, 1105-1113.

(10) Sharif, S.; Ai, J.; Azami, M.; Verdi, J.; Atlasi, M. A.; Shirian, S.; Samadikuchaksaraei, A., Collagen-coated nano-electrospun PCL seeded with human endometrial stem cells for skin tissue engineering applications. $J$. Biomed. Mater. Res. B. Appl. Biomater. 2017, DOI: 10.1002/jbm.b.33966.

(11) Cai, E. Z.; Teo, E. Y.; Jing, L.; Koh, Y. P.; Qian, T. S.; Wen, F.; Lee, J. W.; Hing, E. C.; Yap, Y. L.; Lee, H.; Lee, C. N.; Teoh, S. H.; Lim, J.; Lim, T. C., Bio-conjugated polycaprolactone membranes: a novel wound dressing. Arch. Plast. Surg. 2014, 41, 638-646.

(12) Nhi, T. T.; Khon, H. C.; Hoai, N. T.; Bao, B. C.; Quyen, T. N.; Van Toi, V.; Hiep, N. T., Fabrication of electrospun polycaprolactone coated withchitosansilver nanoparticles membranes for wound dressing applications. J. Mater. Sci. Mater. Med. 2016, 27, 156.

(13) Fair, R. J.; Tor, Y., Antibiotics and bacterial resistance in the 21st century. Perspect. Medicin. Chem. 2014, 6, 25-64.

(14) Zimmerli, W., Clinical presentation and treatment of orthopaedic implantassociated infection. J. Intern. Med. 2014, 276, 111-119.

(15) Sutherland, I., Biofilm exopolysaccharides: a strong and sticky framework. Microbiology. 2001, 147, 3-9.

(16) Payne, D. E.; Boles, B. R., Emerging interactions between matrix components during biofilm development. Curr. Genet. 2016, 62, 137-141.

(17) Davey, M. E.; O'Toole G, A., Microbial biofilms: from ecology to molecular genetics. Microbiol Mol Biol Rev 2000, 64, 847-867. 
(18) Donlan, R. M.; Costerton, J. W., Biofilms: survival mechanisms of clinically relevant microorganisms. Clin. Microbiol. Rev. 2002, 15, 167-193.

(19) Donlan, R. M., Biofilms on central venous catheters: is eradication possible? Curr. Top. Microbiol. Immunol. 2008, 322, 133-161.

(20) Kriegel, C.; Arecchi, A.; Kit, K.; McClements, D. J.; Weiss, J., Fabrication, functionalization, and application of electrospun biopolymer nanofibers. Crit. Rev. Food Sci. Nutr. 2008, 48, 775-797.

(21) Noruzi, M., Electrospun nanofibres in agriculture and the food industry: a review. J. Sci. Food Agric. 2016, 96, 4663-4678.

(22) Bridier, A.; Sanchez-Vizuete, P.; Guilbaud, M.; Piard, J. C.; Naitali, M.; Briandet, R., Biofilm-associated persistence of food-borne pathogens. Food Microbiol. 2015, 45, 167-178.

(23) Srey, S.; Jahid, I. K.; Ha, S.-D., Biofilm formation in food industries: A food safety concern. Food Control. 2013, 31, 572-585.

(24) Mitik-Dineva, N.; Wang, J.; Truong, V. K.; Stoddart, P.; Malherbe, F.; Crawford, R. J.; Ivanova, E. P., Escherichia coli, Pseudomonas aeruginosa, and Staphylococcus aureus attachment patterns on glass surfaces with nanoscale roughness. Curr. Microbiol. 2009, 58, 268-273.

(25) Yuan, Y.; Hays, M. P.; Hardwidgeb, P. R.; Kim, J., Surface characteristics influencing bacterial adhesion to polymeric substrates. RSC $A d v$. 2017, 7, 14254-14261.

(26) Kargar, M.; Ji, W.; Amrinder, N. S.; Bahareh, B., Controlling bacterial adhesion to surfaces using topographical cues: a study of the interaction of Pseudomonas aeruginosa with nanofiber-textured surfaces. Soft Matter. 2012, 40, 10254-10259.

(27) Abrigo, M.; Kingshott, P.; McArthur, S. L., Electrospun polystyrene fiber diameter influencing bacterial attachment, proliferation, and growth. ACS Appl. Mater. Interfaces. 2015, 7, 7644-7652.

(28) Abrigo, M.; Kingshott, P.; McArthur, S. L., Bacterial response to different surface chemistries fabricated by plasma polymerization on electrospun nanofibers. Biointerphases. 2015, 10, 04a301.

(29) Peleg, A. Y.; Seifert, H.; Paterson, D. L., Acinetobacter baumannii: emergence of a successful pathogen. Clin. Microbiol. Rev. 2008, 21, 538-582.

(30) Lister, P. D.; Wolter, D. J.; Hanson, N. D., Antibacterial-resistant Pseudomonas aeruginosa: clinical impact and complex regulation of chromosomally encoded resistance mechanisms. Clin Microbiol Rev 2009, 22, 582-610.

(31) NicAogain, K.; O'Byrne, C. P., The Role of Stress and Stress Adaptations in Determining the Fate of the Bacterial Pathogen Listeria monocytogenes in the Food Chain. Front. Microbiol. 2016, 7, 1865.

(32) Di Domenico, E. G.; Farulla, I.; Prignano, G.; Gallo, M. T.; Vespaziani, M.; Cavallo, I.; Sperduti, I.; Pontone, M.; Bordignon, V.; Cilli, L.; De Santis, A.; Di Salvo, F.; Pimpinelli, F.; Lesnoni La Parola, I.; Toma, L.; Ensoli, F., Biofilm is a Major Virulence Determinant in Bacterial Colonization of Chronic Skin Ulcers Independently from the Multidrug Resistant Phenotype. Int. J. Mol. Sci. 2017, 18. DOI: 10.3390/ijms 18051077.

(33) Branda, S. S.; Vik, S.; Friedman, L.; Kolter, R., Biofilms: the matrix revisited. Trends Microbiol. 2005, 13, 20-26.

(34) Merritt, J. H.; Kadouri, D. E.; O'Toole, G. A., Growing and analyzing static biofilms. Curr. Protoc. Microbiol. 2005, Chapter 1, Unit 1B.1. 
(35) Donlan, R. M., Biofilms: microbial life on surfaces. Emerg. Infect. Dis. 2002, $8,881-890$. 


\section{Graphical abstract}

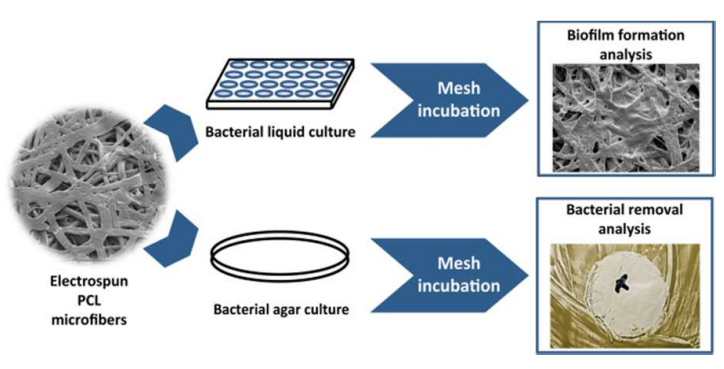

